\title{
Current and emerging management options for patients with Morquio A syndrome
}

This article was published in the following Dove Press journal:

Therapeutics and Clinical Risk Management

8 February 2013

Number of times this article has been viewed

\section{Mohamed F Algahim \\ G Hossein Almassi}

Division of Cardiothoracic Surgery, Medical College of Wisconsin, Milwaukee, WI, USA
Correspondence: G Hossein Almassi Division of Cardiothoracic Surgery, Medical College of Wisconsin, $9200 \mathrm{~W}$ Wisconsin Avenue, East Clinic Building, Milwaukee, WI 53226, USA Tel + I 4149556919

Fax +I 4149666204

Email halmassi@mcw.edu
Abstract: Morquio A syndrome is a lysosomal storage disease associated with mucopolysaccharidosis. It is caused by a deficiency of the lysosomal enzyme, $\mathrm{N}$-acetylgalactosamine6-sulfate sulfatase, which leads to accumulation of keratan sulfate and condroitin-6 sulfate in multiple organs. Patients present with multisystemic complications involving the musculoskeletal, respiratory, cardiovascular, and digestive systems. Presently, there is no definitive cure, and current management options are palliative. Enzyme replacement therapy and hematopoietic stem cell therapy have been proven effective in certain lysosomal storage diseases, and current investigations are underway to evaluate the effectiveness of these therapies and others for the treatment of Morquio A syndrome. This review discusses the current and emerging treatment options for Morquio A syndrome, citing examples of the treatment of other mucopolysaccharidoses.

Keywords: lysosomal, storage disease, mucopolysaccharidosis

\section{Introduction}

Mucopolysaccharidoses (MPSs) are inherited lysosomal storage disorders caused by enzymatic defects in the catabolism of glycosaminoglycans (GAGs). Presently, there are eleven different enzymatic defects associated with seven different types of MPS. The lack of enzymatic activity leads to tissue-specific intracellular accumulation of substrates. Clinically, patients present with multisystemic complications associated with organ-specific dysfunction secondary to the intracellular substrate accumulation.

In 1929, Luis Morquio, of Uruguay, described four family members with features of dysostosis multiplex, corneal clouding, aortic valve disease, and urinary excretion of keratan sulfate. ${ }^{1}$ MPS type IV, also known as Morquio syndrome, is an autosomal recessive disorder and is subclassified into type IV-A (MPS IV-A, Morquio A) and type IV-B (MPS IV-B). Morquio type IV-A is caused by a defect in N-acetylgalactosamine-6-sulfate sulfatase (GALNS; EC 3.1.6.4) - a lysosomal enzyme essential for catabolism of keratan sulfate (KS) and condroitin-6-sulfate (C6S); MPS IV-B is caused by $\beta$-galactosidase-1 deficiency essential for the catabolism of glyconjugates with terminal $\beta$-galactosyl residues. Recent molecular analysis demonstrated that most of MPS IV-A cases result from misfolding of GALNS. ${ }^{2}$ The inability to catabolize GAGs, such as KS and C6S, results in their accumulation within the lysosomes and subsequent cellular and organ dysfunction. As a consequence, patients with Morquio syndrome present with progressive complications specific to cellular involvement of osseous, corneal, valvular, and other organ-specific tissue.

The incidence of MPS IV-A in the United States has not been established. In British Columbia, Canada, the estimates are 1 per 200,000 live births, and in Europe, 
the incidence varies from 1 per 76,000 in Northern Ireland to 1 in 450,000 live births in The Netherlands and Portugal. ${ }^{3-5}$ Neonatal screening for lysosomal storage diseases will allow for better quantification of the incidence of MPS IV-A.

Patients with MPS IV-A appear normal at birth, but initial presenting symptoms often manifest after 1 year of age. ${ }^{6}$ Musculoskeletal complications are the most common presenting features of MPS IV-A; patients may also develop complications involving the cardiac, respiratory, and digestive systems. Unlike most other MPS conditions, MPS IV-A does not affect neurologic function, and patients maintain normal intellect, although behavioral problems with anxiety, depression, and attention have been associated with MPS IV-A. ${ }^{7}$ Morbidity can vary from mild to severe disease those with severe disease present at an earlier age and have more pronounced and rapidly progressive comorbidities, while patients with the attenuated form have a slower rate of progression. Yet the cumulative effect of the disease progression in the mild cases leads to debilitating comorbidities by adulthood. Patients with severe disease die in the second or third decade, while mild disease allows for life into the seventh decade; most patients die of pulmonary infections, cervical instability, and valvular heart disease. ${ }^{8}$

There is no definitive cure for Morquio syndrome; the current standard of care is medical and surgical management of the involved systems with the goal of palliation, prevention, and slowing of the progression of complications. ${ }^{6,8}$ Once diagnosed, treatment of Morquio syndrome requires a system-specific and multidisciplinary approach, often involving primary care physicians, orthopedists, pulmonologist, cardiologists, and anesthesiologists. Other MPS and lysosomal storage diseases - Hurler syndrome (MPS I), Hunter syndrome (MPS II), Maroteaux-Lamy syndrome (MPS VI), Gaucher disease, Fabry disease, and Pompe disease - are currently being treated with enzyme replacement therapy (ERT). Hematopoietic stem cell therapy (HSCT) has played an important role in the treatment of Hurler syndrome and is currently under intensive study for the treatment of other MPSs. The role of ERT and HSCT for the treatment of MPS IV-A is also under investigation.

The present review discusses the complications of MPS IV-A and reviews the current and emerging treatment options.

\section{Complications and current management options}

The musculoskeletal system is often first to be afflicted and involves the appendicular and axial skeletons.
Radiologic evidence of osseous involvement can be diagnostic as early as 6 months of age and often precedes physical manifestation of MPS IV-A. ${ }^{8,9}$ Obvious orthopedic involvement often becomes evident between 2 and 3 years of age, and most patients are diagnosed by 5 years of age. ${ }^{6}$ Patients often present with weakness, stunted growth, dwarfism, pectus carinatum, and genu valgum. Ligamentous laxity and direct bone involvement lead to a delay in ability to walk, limb instability, and a waddling gait. These findings result from accumulation of KS within bone, cartilage, and ligaments that lead to defects in tissue formation. As the disease progresses, patients develop more serious orthopedic complications. The most serious musculoskeletal manifestation of MPS IV-A is odontoid dysplasia and C1-2 instability, which is found in all patients with MPS IV-A. This predisposes to atlantoaxial subluxation with cervical cord compression and progressive myelopathy that leads to gradual or abrupt neurologic deficits, such as muscle weakness, cervical myelopathy, bowel and bladder dysfunction, hemiplegia, quadriparesis, and death. ${ }^{10}$ The risk of cervical spinal cord compression poses a dilemma when the need for intubation arises in patients with cervical instability, as there is a significant risk of iatrogenic damage to the cervical cord at the time of intubation. The risk is further compounded as most patients require multiple surgeries and undergo major surgical interventions by the age of 10 , with the neck being the most common musculoskeletal surgical site. ${ }^{6,11}$ Magnetic resonance imaging is used to screen for involvement of the cervical spine and prophylactic occipito-cervical fusion has been recommended to prevent the associated complications. ${ }^{12-14}$

Difficulty in perioperative airway control is further compounded by previous cervical fusion, chest habitus, short neck, and GAG deposition in the tissues of the airway. ${ }^{15}$ These factors may also delay extubation and predispose patients to tracheostomy. A thorough preoperative evaluation and plan, involving an anesthesiologist experienced with MPS, are essential ahead of surgical intervention requiring intubation. ${ }^{15-17}$ In our experience with an adult MPS IV-A patient requiring intubation for surgery, awake oral fiberoptic intubation was necessary due to diffuse abnormalities of the vertebral bodies and disc spaces, and thoracolumbar kyphosis and dextroscoliosis. ${ }^{18}$ Aside from the aforementioned issues involving operative airway management, involvement of the respiratory system in MPS IV-A predisposes patients to serious complications. Excessive KS deposition in the airway tissue, along with kyphoscoliosis, leads to progressive and debilitating restrictive and obstructive lung processes. ${ }^{8}$ Patients with MPS IV-A develop airway obstruction and sleep 
apnea and are prone to frequent upper respiratory tract infections and pneumonia, which can be a source of significant morbidity. ${ }^{6,8}$ Formal sleep studies should be undertaken to evaluate the severity of sleep apnea; some patients require continuous positive airway pressure devices for obstructive sleep apnea, and home oxygen may be necessary in severe disease. Breathing exercises should also be encouraged. In patients with frequent respiratory infections, seasonal influenza vaccine may be offered, and a low threshold for starting antibiotic regimen when infection is evident is justified; tonsillectomy and adenoidectomy are often indicated in most patients to further prevent infections. ${ }^{6,8,19}$

Cardiovascular complications are common in MPS and can affect the cardiac valves, the coronary arteries, and the aorta. ${ }^{20-22}$ Excessive GAG accumulation in valve, coronary, and aortic tissue leads to thickening of the tissue and subsequent valvular dysfunction, coronary artery intimal sclerosis, and weakening of the aortic wall. Specifically, the accumulation of GAG within tissue is associated with activation of toll-like receptors and inflammatory pathways that likely contribute to the associated cardiovascular complications that predispose patients to valvular stenosis or insufficiency, ischemic heart disease, and aortic aneurysms. ${ }^{20-23}$ These processes are progressive and patients present with symptomatic disease in adulthood. Valvular disease is a common complication in MPS; the left-sided heart valves are affected more than right-sided heart valves, and the mitral valve is most often involved..$^{20,24}$ Surgical correction of symptomatic valvular disease is warranted but can be complicated, and at times avoided because the severe comorbidities associated with the late presentation impart significant and cumulative surgical risks. In MPS IV-A, the aortic valve is most often involved, although the mitral valve is also frequently involved; the pathophysiology is similar to that of other MPSs and is secondary to excessive $\mathrm{KS}$ deposition in the valve tissue and likely activation of inflammatory processes. ${ }^{23,25,26} \mathrm{We}$, amongst others, were successful in aortic valve replacement in a patient with Morquio syndrome. ${ }^{18,27,28}$

Involvement of other organ systems in patients with MPS IV-A predisposes to non-life threatening, but significant, morbidities. Patients develop visual disturbances from excessive GAG deposition in corneal tissue. This leads to corneal clouding with increased light scatter and photophobia; wearing darkened glasses and peaked cap are recommended to alleviate the symptoms. ${ }^{8}$ Hearing loss is common in MPS IV-A. Several factors contribute to the severity of hearing loss, which is due to both conductive and neurosensory deficits. Symptoms of hearing loss are associated dysostosis of the auditory bones and recurring middle ear infections that cause scarring and abnormalities in the inner and middle ears. $^{8,19}$ Patients with severe recurring ear infections may benefit from early placement of ventilating tubes, although many patients ultimately require hearing aids. ${ }^{8,19}$ Patients are also predisposed to dental problems. MPS IV-A patients have unique dental features with small and wide-spaced teeth, spade-shaped incisors, and thin and weak enamel. Patients are predisposed to frequent caries and require meticulous hygiene; prophylactic antibiotics for bacterial endocarditis are necessary in patients requiring dental treatment.

\section{Emerging treatment options ERT}

The concept of ERT was first described in 1964 by Christian de Duve when he speculated, “... any substance which is taken up intracellularly in an endocytic process is likely to end up within lysosomes. This obviously opens up many possibilities for interaction, including replacement therapy."${ }^{9,30}$ In-vivo and in-vitro studies have demonstrated hydrolysis of sucrose in acid-maltase deficient cells of Pompe disease treated with the enzyme interlase. ${ }^{29,31}$ This corroborated the idea of de Duve and led to other important studies in the early development of ERT. The concept of lysosomal storage diseases was new at the time of these studies, and significant progress was made after the identification and purification of the deficient enzymes of several lysosomal storage diseases. ${ }^{29}$ Aside from Pompe disease, many of the early studies were of Hurler syndrome, Hunter syndrome, inclusion cell disease, and Gaucher type 1. Further progress was made after successful identification of the recognition signal essential for intracellular uptake of the deficient enzymes. This was demonstrated with glucocerebrosidase deficiency in Gaucher type I, which became the first lysosomal storage disease to be treated with ERT. Of the MPSs, the first to be treated with ERT was Hurler syndrome (MPS I) using recombinant human $\alpha$-L-iduronidase. Presently, ERT is being used to treat patients with MPS I, MPS II, MPS VI, Fabry, Gaucher, and Pompe disease. ERT for the treatment of MPS IV-A is currently under investigation.

ERT is a lifetime therapy that involves regular intravenous infusions of the recombinant enzyme. Therapy is often associated with infusion reactions that vary from headache, flushing, fever, and/or urticaria to potentially life threatening anaphylactic reactions. Such reactions are due to the development of antibodies against the recombinant enzyme; the incidence may increase concomitantly with the increase in dosage ${ }^{32-36}$ If such reactions become limiting, patients may 
require pretreatment with anti-pyretics and/or anti-histamines in order to prevent severe anaphylactoid reactions; patients may be able to be desensitized over time as well. ${ }^{32}$ Therapy is therefore given in a controlled hospital setting, although a home infusion regimen has been described as a feasible and safe alternative for some patients. ${ }^{32}$

ERT has demonstrated substantial improvements in disease-related comorbidities of MPSs, but ERT does not cross the blood-brain barrier, and the effects on neurocognitive and developmental deterioration have been suboptimal. In patients with Hurler syndrome, treatment with recombinant human $\alpha$-L-iduronidase results in decreased lysosomal storage in the liver and significant improvement in hepatosplenomegaly; improvement in maximal range of shoulder flexion and elbow extension as well as improvement in ambulation; and improvement in sleep apnea. ${ }^{32,34}$ In Hunter syndrome, compared with placebo, patients treated with recombinant human iduronate-2-sulfatase were able to walk greater distances on the 6-minute walk test, had an increase in the percentage of predicted forced vital capacity, and an increase in absolute vital capacity. ${ }^{32,35}$ ERT was approved for Hunter syndrome in the United States and Europe in 2006 and in Japan in 2007. Similar improvements have also been demonstrated in patients treated with human recombinant arylsulphatase B for Maroteaux-Lamy syndrome. These patients experienced clinical improvement in walking and stair climbing, and improved joint range of motion; patients also demonstrated improvement in pulmonary function. . $^{32,35-37}$ ERT in the treatment of these MPSs demonstrated a notable decrease in urinary GAG levels. ERT has not demonstrated significant improvements in neurologic deterioration in MPS patients. In all three MPSs treated with ERT, patients developed some degree of infusion-associated reaction and antibody formation to the recombinant enzyme; although, patients with severe Maroteaux-Lamy syndrome tolerated higher doses of recombinant enzyme therapy and had greater clinical gains. ${ }^{38}$ Furthermore, in sibling case studies of all three of the above MPSs treated with ERT, improved response and benefits in clinical outcomes were demonstrated in the younger siblings diagnosed at birth and started on ERT in the first 6 months of life..$^{32,39-42}$ These findings highlight the importance of early diagnosis and associated positive response to early treatment of MPS.

Continued understanding of ERT and the disease process of the lysosomal storage diseases has set the stage for further application of the treatment in other MPSs. ERT for MPS IV-A is ideal given the lack of neurologic deterioration in these patients. Current investigations to isolate a suitable recombinant GALNS for replacement therapy in humans are underway, and preliminary results in animal models are promising. A knockout mouse model of MPS IV-A has been successful; and GALNS enzyme has been produced and purified using Chinese hamster ovary (CHO) cells and is a source of selectively secreted human recombinant enzyme. ${ }^{43}$ Another potential source of purified recombinant GALNS enzyme under investigation is that derived from Escherichia coli. ${ }^{43,44}$ These findings have allowed for in-vivo studies of ERT and have set the stage for clinical studies in humans. In an in-vivo study of an MPS IV-A mouse model, with 12 weeks of intravenous treatments with two recombinant human GALNSs produced in $\mathrm{CHO}$ cell lines, there was improved lysosomal storage in visceral organs, heart valves, ligaments, and connective tissue; there was also a dosedependent clearance of storage tissue in the brain, and normalization of blood KS levels. ${ }^{45}$ Although ERT is considered unable to cross the blood-brain barrier, the dose-dependent improvement in neurologic response to ERT in the mouse model has been observed by others and is associated with longer duration of treatment. ${ }^{46-48}$ In humans, much of the studies of ERT are early in the investigative process, and data are yet to be published. A human recombinant enzyme, BMN 110 (BioMarin Pharmaceuticals Inc, Novato, CA, USA), is currently under investigation for use in humans. A Phase I/II human multicenter, open-label, dose-escalation study to evaluate safety, tolerability, and efficacy of BMN 110 in patients with MPS IV-A has been completed, and results have yet to be published (NCT00884949). ${ }^{49}$ Presently, there are several ongoing multicenter and multinational studies to investigate the effects of BMN 110 on MPS IV-A patients, and include: a Phase II study specific to patients with limited ambulation (NCT01697139); a randomized, double-blind, pilot study assessing the safety and physiologic effects of BMN 110 (NCT01609062); and several studies to assess long-term effects and safety of BMN 110 (NCT01242111, NCT01415427, NCT01275066), including those less than 5 years of age (NCT01515956) ${ }^{50-53}$ Furthermore, the response to BMN 110 therapy, and potentially other recombinant enzymes, was evaluated in a study of biomarkers of MPS IV-A; alpha-1-antitrypsin, lipoprotein(a), and serum amyloid $\mathrm{P}$ were reported as suitable candidate biomarkers, in addition to $\mathrm{KS} .{ }^{54}$

While much work has yet to be done assessing ERT for the treatment of MPS IV-A, substantial progress has been made. The outcomes of the aforementioned clinical trials will dictate the role of ERT as a treatment option for MPS IV-A in the near future. 


\section{HSCT}

The basis of HSCT for the treatment of MPS and other inherited metabolic disorders was established in 1968 when Fratantoni, Hall, and Neufeld demonstrated correction of biochemical defects of skin fibroblasts from patients with Hunter and Hurler syndromes when these cells were mixed with each other or normal cells. ${ }^{55}$ Later studies with transfusion of plasma and leukocytes demonstrated improvement in degradation of GAGs in patients with Hurler and Hunter syndromes. ${ }^{56,57}$ In 1980, bone marrow transplant was successfully performed in a 1-year-old boy with Hurler syndrome (described below). ${ }^{58}$

HSCT entails transplantation of multipotent hematopoietic stem cells derived from bone marrow, peripheral blood, or umbilical cord blood from a healthy donor to a patient with innate cellular dysfunction to correct the dysfunctional cell line and the associated disease process. Appropriate human leukocyte antigen matching is essential for allogeneic graft transplantation, and complete ablation of the recipient's immune system is necessary. This predisposes patients to complications of immune deficiency and serious graftversus-host disease reactions. In spite of improved methods of stem-cell matching, HSCT remains a high risk procedure with substantial morbidity and mortality; therapy is generally reserved for patients with severe phenotype. When considering HSCT, established practice guidelines should be followed and a multidisciplinary approach should be undertaken to ensure optimal benefit and minimal risk of therapy, with the goal of long-term survival and improved quality of life. ${ }^{59,60}$

HSCT is an evolving alternative for the treatment of MPS. Ongoing replacement of the deficient lysosomal enzymes is achieved by transplanting the enzyme-deficient cell line with enzyme-competent donor cells capable of gaining access to the affected tissue, including the central nervous system. When compared with ERT, HSCT demonstrated superior reduction in substrate burden, and has been shown to prevent and/or cure associated musculoskeletal and organ-specific complications; unlike ERT, HSCT's ability to access the central nervous system allows for treatment of neurocognitive degeneration. ${ }^{61}$ In the first successful bone marrow transplant of a patient with MPS, a 1-year-old boy with Hurler syndrome, the patient developed acute graft-versus-host disease, but 13 months after transplant, there was reversal of hepatosplenomegaly and corneal clouding, leukocyte iduronidase activity increased to that of a heterozygote, and arrest in neurocognitive and developmental deterioration was notable..$^{58,62}$ The preservation of neurocognitive and intellectual development in patients with Hurler syndrome treated with HSCT has been established with more recent studies, and is considered one of the most important benefits of HSCT ${ }^{62-64}$ Other benefits of HSCT in Hurler syndrome include: improvement in hearing, joint mobility, respiratory function, and cardiac function. ${ }^{62}$ Given the associated high risk of morbidity and mortality of HSCT, the current guideline recommendations of the International Consensus Panel on the Management and Treatment of Mucopolysaccharidosis I indicate a multidisciplinary approach on the decision to pursue HSCT; and HSCT must be performed early in the disease course - less than 2 years of age and before developmental deterioration begins - and is limited to patients with an intelligence quotient of $\geq 70 \%{ }^{62}$

While the effects of HSCT on Hurler syndrome are well established, HSCT for the treatment of other MPSs, particularly the effect on the neurologic deficits, has yet to be fully elucidated. The delayed presentation of other MPSs and the subsequent late initiation of therapy have been associated with the suboptimal effects of HSCT on neurologic function in certain MPSs. In patients with Hunter syndrome, HSCT demonstrated improvement in visceral and soft tissue involvement, but the effects on neurologic symptoms has not been substantial. Although, in a retrospective study of 21 patients with Hunter syndrome who received HSCT, 9.6 years after treatment, activities of daily living were maintained, improvements in cribriform changes and brain ventricular dilatation were noted, and stabilization of brain atrophy was also noted ${ }^{65}$ Consistent with the idea that early diagnosis and initiation of treatment are important, the authors conclude that the effect of HSCT on the brain is optimized by treatment prior to clinical manifestations of developmental delay; and the poor response to HSCT is associated with severity of the syndrome. Investigational data of HSCT for the other MPSs are few, and results for neurocognitive benefits are mixed. ${ }^{32}$ In patients with Sanfilippo syndrome, HSCT has stabilized disease but with less impact on cognition; in two patients transplanted under the age of 2 years, modest cognitive gains were noted with improvement in behavior and sleeping patterns 3-5 years post transplant. ${ }^{59,66}$ Patients with Maroteaux-Lamy syndrome have reduced life expectancy and are candidates for HSCT; therapy has demonstrated improvements in hepatosplenomegaly, cardiopulmonary function, visual acuity, and mobility. ${ }^{59,67}$

The role of HSCT in MPS IV-A is currently investigational. In a 2012 report by the Agency for Healthcare Research and Quality (Rockville, MD, USA) on the status of HSCT for Morquio syndrome and other childhood diseases, it was 
concluded that "the strength of the body of evidence is insufficient to draw conclusions on the comparative benefit of single HSCT compared with symptom management and or disease natural history with respect to neurocognitive and neurodevelopmental outcomes for MPS IV-A." ${ }^{68}$ As it relates to MPS IV-A, HSCT has not been shown to substantially treat the severe skeletal manifestation; this is likely due to lower vascularization of bone tissue. But, the improvement and reversal of the somatic complications of other MPSs treated with HSCT substantiate consideration of HSCT for the treatment of the hepatic, cardiovascular, respiratory, and digestive complications of MPS IV-A. The use of HSCT in MPS is limited to those with a severe phenotype with neurologic involvement. Although HSCT has demonstrated superiority to ERT, its use is limited by a poor safety profile. ${ }^{61}$ Consequently, clinical data of HSCT in milder phenotypes and in MPS where neurologic function is preserved - including MPS IV-A - are few and limited. Recent data have demonstrated improved survival with HSCT, making the case for the use of HSCT in milder phenotypes to allow for further investigations and understanding of treatment. ${ }^{69,70}$ While few data exist for HSCT in MPS IV-A, successful bone marrow transplantation was tolerated, with excellent survival, in patients with MPS IV-A transplanted for sickle cell anemia. ${ }^{71,72}$ In a recent description of a male patient with MPS IV-A treated with HSCT at an advanced age, 5 years after successful allogeneic bone marrow transplantation, the patient demonstrated recovery of GALNS activity in lymphocytes and improvements of motor function, respiratory function, and glaucoma; there was also an increase of bone mineral density and an overall improvement in quality of life. ${ }^{8}$ These findings suggest there is much to be learned about HSCT; as our understanding expands, HSCT may play a future role in the treatment of MPS IV-A. Further investigations are warranted to address the issues of safety of therapy, optimal time for transplantation, donor type, and other factors to ensure successful engraftment, and the role of HSCT in milder phenotypes of MPS.

\section{Gene therapy}

HSCT and ERT have shown promise for the treatment of MPS IV-A and other MPSs. Current alternative and potential adjunct therapies are under investigation to address the limitations of HSCT and ERT; particularly, the limited effects on the central and musculoskeletal systems. One such therapy includes gene therapy; we briefly discuss this treatment option for MPS IV-A.

The goal of gene therapy is to correct the genetic defect by direct insertion of normal DNA into the affected cells to institute endogenous production of the deficient enzyme by these cells. Current methods include using viral vectors to directly treat affected tissue or using inherent cellular properties for cross-correction. Direct administration involves targeted gene therapy vectors into isolated organ tissue, such as the brain, to target specific dysfunctional organ tissue. Cross-correction employs the same principles of ERT and that of the early studies of HSCT, as affected cells take up the target enzyme produced and leaked by cells of another organ system treated with gene therapy, eg, the liver. These methods have demonstrated promising results in animal models of lysosomal storage diseases. Directly administering gene therapy via recombinant adeno-associated virus vector into the diaphragm of a mouse model of Pompe disease has resulted in improved diaphragmatic muscle and respiratory function. ${ }^{73,74}$ In another murine model of Pompe disease, liver-directed recombinant adeno-associated virus vectors demonstrated cross-correction of skeletal and cardiac muscle with improved function and glycogen storage. ${ }^{75,76}$ Gene therapy may also play a role as an adjunct to HSCT, as autologous bone marrow or hematopoietic stem cells are treated with gene therapy to express the target enzyme prior to transplantation. ${ }^{77}$ These findings are promising, but much work is yet to be done to address the issues of immune reaction, choice of vector, and optimal route of administration of gene therapy.

\section{Conclusion}

Morquio A syndrome is a lysosomal storage disease with severe musculoskeletal complications. Symptoms are progressive and involve other organ systems, including the heart, respiratory, and visceral organs. Presently, treatment is palliative and focused on alleviation of organ-specific complications. While the role of HSCT and gene therapy for the treatment of MPS IV-A has yet to be fully defined with further animal and human studies, the current data of ERT are promising. These findings suggest ERT will likely play a key role in the future treatment of MPS IV-A.

\section{Disclosure}

The authors report no conflicts of interest in this work.

\section{References}

1. Morquio L. Sur une forme de dystrophie osseuse familiale. Archives de Médecine des Infants. 1929;32:129-135. French.

2. Rivera-Colon Y, Schutsky EK, Kita AZ, Garman SC. The structure of human GALNS reveals the molecular basis for mucopolysaccharidosis IVA. J Mol Biol. 2012;423(5):736-751.

3. Applegarth DA, Toone JR, Lowry RB. Incidence of inborn errors of metabolism in British Columbia, 1969-1996. Pediatrics. 2000;105(1):e10. 
4. Nelson J. Incidence of the mucopolysaccharidoses in Northern Ireland. Hum Genet. 1997;101(3):355-358.

5. Poorthuis BJ, Wevers RA, Kleijer WJ, et al. The frequency of lysosomal storage diseases in The Netherlands. Hum Genet. 1999; 105(1-2):151-156.

6. Montano AM, Tomatsu S, Gottesman GS, Smith M, Orii T. International Morquio A Registry: clinical manifestation and natural course of Morquio A disease. J Inherit Metab Dis. 2007;30(2):165-174.

7. Davison JE, Kearney S, Horton J, Foster K, Peet AC, Hendriksz CJ. Intellectual and neurological functioning in Morquio syndrome (MPS IVa). J Inherit Metab Dis. Epub January 10, 2012.

8. Tomatsu S, Montano AM, Oikawa H, et al. Mucopolysaccharidosis type IVA (Morquio A disease): clinical review and current treatment. Curr Pharm Biotechnol. 2011;12(6):931-945.

9. Langer LO Jr, Carey LS. The roentgenographic features of the KS mucopolysaccharidosis of Morquio (Morquio-Brailsford's disease). Am J Roentgenol Radium Ther Nucl Med. 1966;97(1):1-20.

10. Tong CK, Chen JC, Cochrane DD. Spinal cord infarction remote from maximal compression in a patient with Morquio syndrome. J Neurosurg Pediatr. 2012;9(6):608-612.

11. Dhawale AA, Thacker MM, Belthur MV, Rogers K, Bober MB, Mackenzie WG. The lower extremity in Morquio syndrome. J Pediatr Orthop. 2012;32(5):534-540.

12. Oncag G, Ertan Erdinc AM, Cal E. Multidisciplinary treatment approach of Morquio syndrome (mucopolysaccharidosis type IVA). Angle Orthod. 2006;76(2):335-340.

13. Svensson O, Aaro S. Cervical instability in skeletal dysplasia. Report of 6 surgically fused cases. Acta Orthop Scand. 1988;59(1):66-70.

14. Ransford AO, Crockard HA, Stevens JM, Modaghegh S. Occipitoatlanto-axial fusion in Morquio-Brailsford syndrome. A ten-year experience. J Bone Joint Surg Br. 1996;78(2):307-313.

15. Theroux MC, Nerker T, Ditro C, Mackenzie WG. Anesthetic care and perioperative complications of children with Morquio syndrome. Paediatr Anaesth. 2012;22(9):901-907.

16. Walker RW. Management of the difficult airway in children. $J R S o c$ Med. 2001;94(7):341-344.

17. Bartz HJ, Wiesner L, Wappler F. Anaesthetic management of patients with mucopolysaccharidosis IV presenting for major orthopaedic surgery. Acta Orthop Scand. 1999;43(6):679-683.

18. Pagel PS, Almassi GH. Perioperative implications of Morquio syndrome in a 31-year-old woman undergoing aortic valve replacement. J Cardiothorac Vasc Anesth. 2009;23(6):855-857.

19. Northover H, Cowie RA, Wraith JE. Mucopolysaccharidosis type IVA (Morquio syndrome): a clinical review. J Inherit Metab Dis. 1996;19(3):357-365.

20. Braunlin EA, Harmatz PR, Scarpa M, et al. Cardiac disease in patients with mucopolysaccharidosis: presentation, diagnosis and management. J Inherit Metab Dis. 2011;34(6):1183-1197.

21. Engle J, Safi HJ, Abbassi O, et al. Mucopolysaccharidosis presenting as pediatric multiple aortic aneurysm: first reported case. J Vasc Surg. 1997;26(4):704-710.

22. Factor SM, Biempica L, Goldfischer S. Coronary intimal sclerosis in Morquio's syndrome. Virchows Arch A Pathol Anat Histol. 1978; 379(1):1-10.

23. Metcalf JA, Linders B, Wu S, et al. Upregulation of elastase activity in aorta in mucopolysaccharidosis I and VII dogs may be due to increased cytokine expression. Mol Genet Metab. 2010;99(4):396-407.

24. Tan CT, Schaff HV, Miller FA Jr, Edwards WD, Karnes PS. Valvular heart disease in four patients with Maroteaux-Lamy syndrome. Circulation. 1992;85(1):188-195.

25. Diaz JH, Belani KG. Perioperative management of children with mucopolysaccharidoses. Anesth Analg. 1993;77(6):1261-1270.

26. John RM, Hunter D, Swanton RH. Echocardiographic abnormalities in type IV mucopolysaccharidosis. Arch Dis Child. 1990;65(7):746-749.

27. Barry MO, Beardslee MA, Braverman AC. Morquio's syndrome: severe aortic regurgitation and late pulmonary autograft failure. $J$ Heart Valve Dis. 2006;15(6):839-842.
28. Nicolini F, Corradi D, Bosio S, Gherli T. Aortic valve replacement in a patient with morquio syndrome. Heart Surg Forum. 2008; 11(2):E96-E98.

29. Neufeld EF. Enzyme replacement therapy - a brief history. In: Mehta A, Beck M, Sunder-Plassmann G, editors. Fabry Disease: perspectives from 5 years of FOS. Oxford: Oxford PharmaGenesis; 2006.

30. Deduve C. From cytases to lysosomes. Fed Proc. 1964;23:1045-1049.

31. Cohn ZA, Ehrenreich BA. The uptake, storage, and intracellular hydrolysis of carbohydrates by macrophages. J Exp Med. 1969; 129(1):201-225.

32. Valayannopoulos V, Wijburg FA. Therapy for the mucopolysaccharidoses. Rheumatology (Oxford). 2011;50 Suppl 5:v49-v59.

33. Giugliani R, Rojas VM, Martins AM, et al. A dose-optimization trial of laronidase (Aldurazyme) in patients with mucopolysaccharidosis I. Mol Genet Metab. 2009;96(1):13-19.

34. Kakkis ED, Muenzer J, Tiller GE, et al. Enzyme-replacement therapy in mucopolysaccharidosis I. N Engl J Med. 2001;344(3):182-188.

35. Muenzer J, Wraith JE, Beck M, et al. A Phase II/III clinical study of enzyme replacement therapy with idursulfase in mucopolysaccharidosis II (Hunter syndrome). Genet Med. 2006;8(8):465-473.

36. Harmatz P, Giugliani R, Schwartz I, et al. Enzyme replacement therapy for mucopolysaccharidosis VI: a phase 3, randomized, double-blind, placebo-controlled, multinational study of recombinant human $\mathrm{N}$-acetylgalactosamine 4-sulfatase (recombinant human arylsulfatase B or rhASB) and follow-on, open-label extension study. J Pediatr. 2006;148(4):533-539.

37. Harmatz P, Ketteridge D, Giugliani R, et al. Direct comparison of measures of endurance, mobility, and joint function during enzymereplacement therapy of mucopolysaccharidosis VI (Maroteaux-Lamy syndrome): results after 48 weeks in a phase 2 open-label clinical study of recombinant human $\mathrm{N}$-acetylgalactosamine 4-sulfatase. Pediatrics. 2005;115(6):e681-e689.

38. Harmatz P, Whitley CB, Waber L, et al. Enzyme replacement therapy in mucopolysaccharidosis VI (Maroteaux-Lamy syndrome). J Pediatr. 2004;144(5):574-580.

39. McGill JJ, Inwood AC, Coman DJ, et al. Enzyme replacement therapy for mucopolysaccharidosis VI from 8 weeks of age - a sibling control study. Clin Genet. 2010;77(5):492-498.

40. Wang RY, Cambray-Forker EJ, Ohanian K, et al. Treatment reduces or stabilizes brain imaging abnormalities in patients with MPS I and II. Mol Genet Metab. 2009;98(4):406-411.

41. Gabrielli O, Clarke LA, Bruni S, Coppa GV. Enzyme-replacement therapy in a 5-month-old boy with attenuated presymptomatic MPS I: 5-year follow-up. Pediatrics. 2010;125(1):e183-e187.

42. Tylki-Szymanska A, Jurecka A, Zuber Z, Rozdzynska A, Marucha J, Czartoryska B. Enzyme replacement therapy for mucopolysaccharidosis II from 3 months of age: a 3-year follow-up. Acta Paediatr. 2012;101(1):e42-e47.

43. Tomatsu S, Montano AM, Gutierrez M, et al. Characterization and pharmacokinetic study of recombinant human $\mathrm{N}$-acetylgalactosamine6-sulfate sulfatase. Mol Genet Metab. 2007;91(1):69-78.

44. Rodriguez A, Espejo AJ, Hernandez A, et al. Enzyme replacement therapy for Morquio A: an active recombinant $\mathrm{N}$-acetylgalactosamine6-sulfate sulfatase produced in Escherichia coli BL21. J Ind Microbiol Biotechnol. 2010;37(11):1193-1201.

45. Tomatsu S, Montano AM, Ohashi A, et al. Enzyme replacement therapy in a murine model of Morquio A syndrome. Hum Mol Genet. 2008;17(6):815-824.

46. Dunder U, Kaartinen V, Valtonen P, et al. Enzyme replacement therapy in a mouse model of aspartylglycosaminuria. FASEB $J$. 2000;14(2):361-367.

47. Vogler C, Levy B, Grubb JH, et al. Overcoming the blood-brain barrier with high-dose enzyme replacement therapy in murine mucopolysaccharidosis VII. Proc Natl Acad Sci U S A. 2005;102(41):14777-14782.

48. Roces DP, Lullmann-Rauch R, Peng J, et al. Efficacy of enzyme replacement therapy in alpha-mannosidosis mice: a preclinical animal study. Hum Mol Genet. 2004;13(18):1979-1988. 
49. BioMarin Pharmaceuticals Inc. A Phase I/II, Multicenter, Open-Label, Dose escalation study to evaluate the safety, tolerability, and efficacy of BMN 110 in subjects with mucopolysaccharidosis type IVA. In: ClinicalTrials.gov [website on the Internet]. Bethesda, MD: US National Library of Medicine; 2009 [updated July 15, 2011]. Available from: http://www.clinicaltrials.gov/ct2/show/NCT00884949. NLM identifier: NCT00884949. Accessed January 7, 2013.

50. BioMarin Pharmaceuticals Inc. A double-blind study to evaluate the efficacy and safety of BMN 110 in patients with mucopolysaccharidosis IVA (Morquio A syndrome). In: ClinicalTrials.gov [website on the Internet]. Bethesda, MD: US National Library of Medicine; 2011 [updated August 6, 2012]. Available from: http://www.clinicaltrials. gov/ct2/show/NCT01275066. NLM identifier: NCT01275066. Accessed January 7, 2013.

51. BioMarin Pharmaceuticals Inc. Long-term efficacy and safety extension study of BMN 110 in patients with mucopolysaccharidosis IVA (Morquio A syndrome). In: ClinicalTrials.gov [website on the Internet]. Bethesda, MD: US National Library of Medicine; 2011 [updated September 18, 2012]. Available from: http://www.clinicaltrials.gov/ ct2/show/NCT01415427. NLM identifier: NCT01415427. Accessed January 7, 2013.

52. BioMarin Pharmaceuticals Inc. Study of BMN 110 in pediatric patients $<5$ years of age with mucopolysaccharidosis IVA (Morquio A syndrome). In: ClinicalTrials.gov [website on the Internet]. Bethesda, MD: US National Library of Medicine; 2011 [updated September 18, 2012]. Available from: http://www.clinicaltrials.gov/ct2/show/ NCT01515956. NLM identifier: NCT01515956. Accessed January 7, 2013.

53. BioMarin Pharmaceuticals Inc. A study to evaluate the long-term efficacy and safety of BMN 110 in patients with mucopolysaccharidosis IVA (Morquio A syndrome). In: ClinicalTrials.gov [website on the Internet]. Bethesda, MD: US National Library of Medicine; 2010 [updated May 10, 2012]. Available from: http://www.clinicaltrials.gov/ ct2/show/NCT01242111. NLM identifier: NCT01242111. Accessed January 7, 2013.

54. Martell L, Lau K, Mei M, Burnett V, Decker C, Foehr ED. Biomarker analysis of Morquio syndrome: identification of disease state and drug responsive markers. Orphanet J Rare Dis. 2011;6:84.

55. Fratantoni JC, Hall CW, Neufeld EF. Hurler and Hunter syndromes: mutual correction of the defect in cultured fibroblasts. Science. 1968;162(3853):570-572.

56. Di Ferrante N, Nichols BL, Donnelly PV, Neri G, Hrgovcic R, Berglund RK. Induced degradation of glycosaminoglycans in Hurler's and Hunter's syndromes by plasma infusion. Proc Natl Acad Sci U S A. 1971;68(2):303-307.

57. Knudson AG Jr, Di Ferrante N, Curtis JE. Effect of leukocyte transfusion in a child with type II mucopolysaccharidosis. Proc Natl Acad Sci U S A. 1971;68(8):1738-1741.

58. Hobbs JR, Hugh-Jones K, Barrett AJ, et al. Reversal of clinical features of Hurler's disease and biochemical improvement after treatment by bone-marrow transplantation. Lancet. 1981;2(8249):709-712.

59. Prasad VK, Kurtzberg J. Transplant outcomes in mucopolysaccharidoses. Semin Hematol. 2010;47(1):59-69.

60. Peters C, Steward CG; National Marrow Donor Program; International Bone Marrow Transplant Registry; Working Party on Inborn Errors, European Bone Marrow Transplant Group. Hematopoietic cell transplantation for inherited metabolic diseases: an overview of outcomes and practice guidelines. Bone Marrow Transplant. Feb 2003;31(4):229-239.

61. Wynn RF, Wraith JE, Mercer J, et al. Improved metabolic correction in patients with lysosomal storage disease treated with hematopoietic stem cell transplant compared with enzyme replacement therapy. J Pediatr. 2009;154(4):609-611.
62. Muenzer J, Wraith JE, Clarke LA; International Consensus Panel on Management and Treatment of Mucopolysaccharidosis I. Mucopolysaccharidosis I: management and treatment guidelines. Pediatrics. Jan 2009;123(1):19-29.

63. Whitley CB, Belani KG, Chang PN, et al. Long-term outcome of Hurler syndrome following bone marrow transplantation. Am J Med Genet. 1993;46(2):209-218.

64. Souillet G, Guffon N, Maire I, et al. Outcome of 27 patients with Hurler's syndrome transplanted from either related or unrelated haematopoietic stem cell sources. Bone Marrow Transplant. 2003; 31(12):1105-1117.

65. Tanaka A, Okuyama T, Suzuki Y, et al. Long-term efficacy of hematopoietic stem cell transplantation on brain involvement in patients with mucopolysaccharidosis type II: a nationwide survey in Japan. Mol Genet Metab. 2012;107(3):513-520.

66. Prasad VK, Mendizabal A, Parikh SH, et al. Unrelated donor umbilical cord blood transplantation for inherited metabolic disorders in 159 pediatric patients from a single center: influence of cellular composition of the graft on transplantation outcomes. Blood. 2008;112(7):2979-2989.

67. Lee V, Li CK, Shing MM, et al. Umbilical cord blood transplantation for Maroteaux-Lamy syndrome (mucopolysaccharidosis type VI). Bone Marrow Transplant. 2000;26(4):455-458.

68. Belinson S, Mauger Rothenberg B, Chopra R, Aronson N. Future Research Needs for Hematopoietic Stem-Cell Transplantation in the Pediatric Population: Identification of Future Research Needs From Comparative Effectiveness Review No 48. Rockville (MD): Agency for Healthcare Research and Quality (US); 2012.

69. Staba SL, Escolar ML, Poe M, et al. Cord-blood transplants from unrelated donors in patients with Hurler's syndrome. $N$ Engl $J$ Med. 2004;350(19):1960-1969.

70. Wynn RF, Mercer J, Page J, Carr TF, Jones S, Wraith JE. Use of enzyme replacement therapy (Laronidase) before hematopoietic stem cell transplantation for mucopolysaccharidosis I: experience in 18 patients. J Pediatr. 2009;154(1):135-139.

71. Mentzer WC, Packman S, Wara W, Morton C. Successful bone marrow transplant in a child with sickle cell anemia and Morquio's disease. Blood. 1990;76:69a

72. Johnson FL, Mentzer WC, Kalinyak KA, Sullivan KM, Abboud MR. Bone marrow transplantation for sickle cell disease. The United States experience. Am J Pediatr Hematol Oncol. 1994;16(1):22-26.

73. Mah CS, Falk DJ, Germain SA, et al. Gel-mediated delivery of AAV1 vectors corrects ventilatory function in Pompe mice with established disease. Mol Ther. 2010;18(3):502-510.

74. Mah C, Fraites TJ Jr, Cresawn KO, Zolotukhin I, Lewis MA, Byrne BJ. A new method for recombinant adeno-associated virus vector delivery to murine diaphragm. Mol Ther. 2004;9(3):458-463.

75. Cresawn KO, Fraites TJ, Wasserfall C, et al. Impact of humoral immune response on distribution and efficacy of recombinant adeno-associated virus-derived acid alpha-glucosidase in a model of glycogen storage disease type II. Hum Gene Ther. 2005;16(1):68-80.

76. Sun B, Zhang H, Bird A, Li S, Young SP, Koeberl DD. Impaired clearance of accumulated lysosomal glycogen in advanced Pompe disease despite high-level vector-mediated transgene expression. J Gene Med. 2009;11(10):913-920.

77. Naldini L. Ex vivo gene transfer and correction for cell-based therapies. Nat Rev Genet. 2011;12(5):301-315. 
Therapeutics and Clinical Risk Management

Dovepress

\section{Publish your work in this journal}

Therapeutics and Clinical Risk Management is an international, peerreviewed journal of clinical therapeutics and risk management, focusing on concise rapid reporting of clinical studies in all therapeutic areas, outcomes, safety, and programs for the effective, safe, and sustained use of medicines. This journal is indexed on PubMed Central, CAS,
EMBase, Scopus and the Elsevier Bibliographic databases. The manuscript management system is completely online and includes a very quick and fair peer-review system, which is all easy to use. Visit http://www.dovepress.com/testimonials.php to read real quotes from published authors.

Submit your manuscript here: http://www.dovepress.com/therapeutics-and-clinical-risk-management-journal 